\title{
The Added Value of Volume-of-Interest C-Arm CT Imaging during Endovascular Treatment of Intracranial Aneurysms
}

\author{
(D) G. Chintalapani, P. Chinnadurai, A. Maier, Y. Xia, S. Bauer, H. Shaltoni, H. Morsi, and M.E. Mawad
}

\begin{abstract}
BACKGROUND AND PURPOSE: Successful endovascular treatment of intracranial aneurysms requires understanding the exact relationship of implanted devices to the aneurysm, parent artery, and other branch vessels during the treatment. Intraprocedural C-arm CT imaging has been shown to provide such information. However, its repeated use is limited due to increasing radiation exposure to the patient. The goal of this study was to evaluate a new volume-of-interest C-arm CT imaging technique, which would provide device-specific information through multiple 3D acquisitions of only the region of interest, thus reducing cumulative radiation exposure to the patient.
\end{abstract}

MATERIALS AND METHODS: VOI C-arm CT images were obtained in 28 patients undergoing endovascular treatment of intracranial aneurysms. VOI images were acquired with the x-ray source collimated around the deployed device, both horizontally and vertically. The images were reconstructed by using a novel prototype robust reconstruction algorithm to minimize truncation artifacts from double collimation. The reconstruction accuracy of VOI C-arm CT images was assessed quantitatively by comparing them with the full-head noncollimated images.

RESULTS: Quantitative analysis showed that the quality of VOI C-arm CT images is comparable with that of the standard Feldkamp, Davis, and Kress reconstruction of noncollimated C-arm CT images (correlation coefficient $=0.96$ and structural similarity index $=0.92$ ). Furthermore, $91.5 \%$ reduction in dose-area product was achieved with VOI imaging compared with the full-head acquisition.

CONCLUSIONS: VOI imaging allows multiple 3D C-arm CT acquisitions and provides information related to device expansion, parent wall apposition, and neck coverage during the procedure, with very low additional radiation exposure to the patient.

ABBREVIATIONS: ATRACT $=$ Approximated Truncation Robust Algorithm for CT; DAP $=$ dose-area product; FDK $=$ Feldkamp, Davis, and Kress; SSIM $=$ Structural Similarity Index Metric

E ndovascular treatment of intracranial aneurysms primarily involves deployment of devices either in the parent artery and/or the aneurysm itself, allowing the aneurysm to thrombose with time. Self-expanding metallic stents, both open-cell and closedcell designs, are routinely used during stent-assisted coiling. It has been shown that asymmetric, malapposed, misaligned, or pro-

Received October 1, 2014; accepted after revision August 20, 2015.

From the Angiography Division (G.C., P.C.), Siemens Medical Solutions USA, Hoffman Estates, Illinois; Pattern Recognition Lab (A.M., Y.X.), Friedrich-Alexander-University, Erlangen-Nuremberg, Germany; Angiography Division (S.B.), Siemens AG, Healthcare Sector, Forchheim, Germany; Neurovascular Center (H.S.), CHI St. Luke's Health System, Houston, Texas; and Department of Radiology (H.M. M.E.M.), Baylor College of Medicine, Houston, Texas.

This study was conducted as part of an investigator-sponsored research study with Siemens Medical Solutions USA.

Abstracts previously presented at: American Society of Neuroradiology Annual Meeting and the Foundation of the ASNR Symposium, April 21-16, 2012; New York, New York; and May 18-23, 2013; San Diego, California.

Please address correspondence to Gouthami Chintalapani, PhD, 2501 North Barrington Rd, Hoffman Estates, IL 60192; e-mail: gouthami.chintalapani@siemens.com http://dx.doi.org/10.3174/ajnr.A4605 lapsed stent elements can result in changes in hemodynamics, leading to subacute or in-stent stenosis. ${ }^{1}$ Thus, obtaining relevant device-specific information such as stent expansion, wall apposition, and aneurysm neck coverage during the treatment is very critical for successful embolization. Similarly, with new-generation flow-diverter devices, inadequate wall apposition, device positioning across the aneurysm neck, and deployment techniques have been shown to influence the amount of metal coverage across the neck, the extent of flow diversion, and the time to form thrombus. ${ }^{2,3}$ Thus, it is very critical to obtain this information during the procedure to evaluate the accuracy of deployment and treatment efficacy, but also to allow additional corrective measures in case of inaccurate deployment.

C-arm CT imaging technology has been shown to provide high-quality CT-like 3D images during interventional procedures that facilitate proper assessment of the deployed device and intraprocedural guidance. ${ }^{4-10}$ Although much of this information can be obtained on 2D fluoroscopic images, desirable material properties of these devices, such as low profile and high flexibility, 


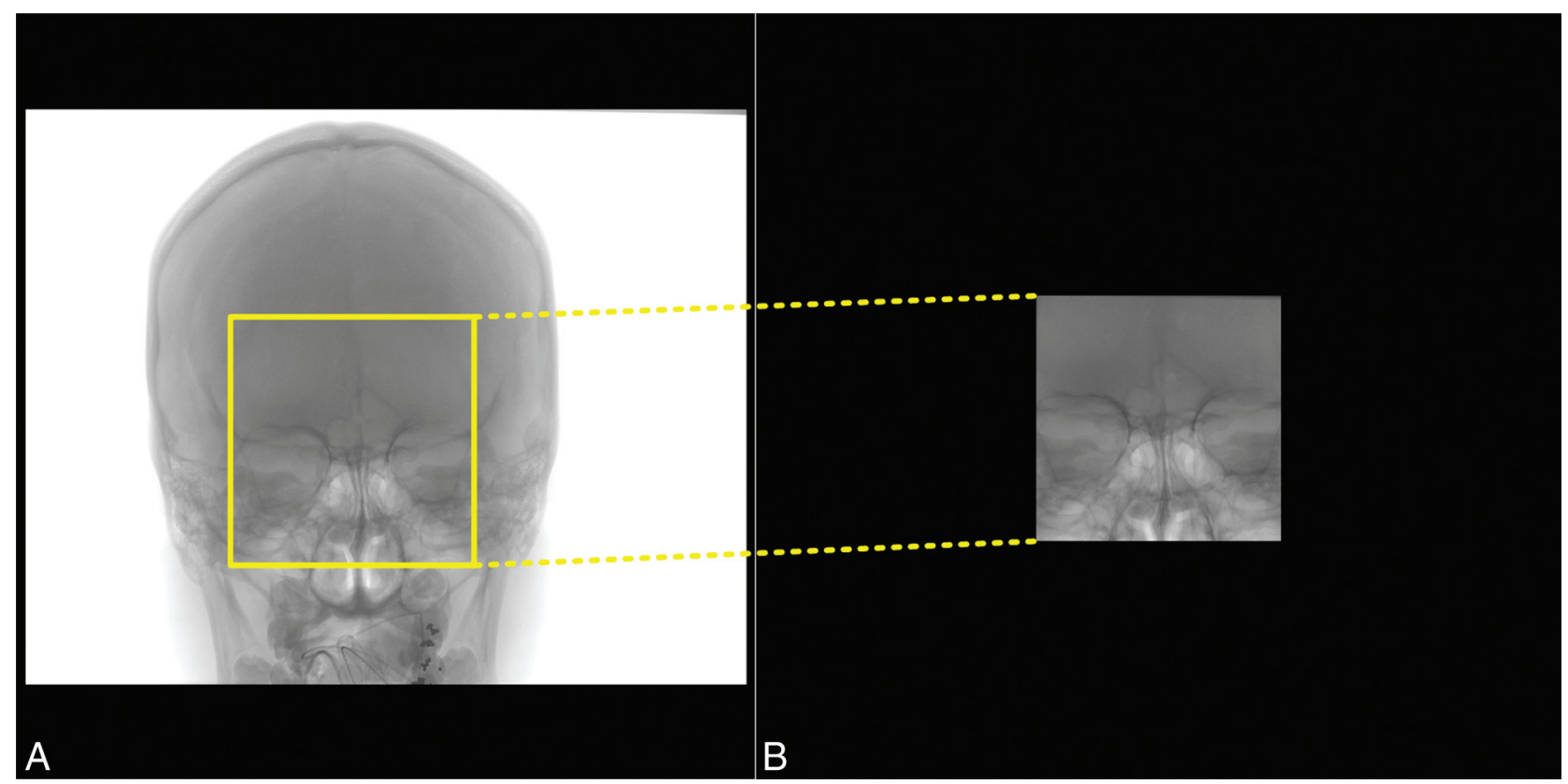

FIG 1. Sample projection image from noncollimated full-head C-arm CT acquisition ( $A$ ) and VOI C-arm CT acquisition (B) with the x-ray source collimated in both horizontal and vertical directions.

often make it difficult to visualize them under conventional 2D angiographic imaging and present a great need for C-arm CT imaging during the intervention. However, acquisition of multiple C-arm CT images is limited because of the added radiation exposure to the patient. The reported effective dose of a typical full-head C-arm CT scan is 2.9 mSv. ${ }^{11}$ Thus, multiple C-arm CT acquisitions in a single treatment session can easily increase the cumulative dose delivered to the patient.

Alternatively, the radiation dose due to the C-arm CT acquisition can be reduced with collimation. Because the physician's focus is on the device and its relationship to the parent artery, the $\mathrm{x}$-ray source can be collimated in both horizontal and vertical directions to image only the ROI and block the radiation exposure outside the ROI (Fig 1). With double collimation, the conventional Feldkamp, Davis, and Kress (FDK) reconstruction algorithm results in severe truncation artifacts because patient anatomy is not fully exposed in all projections, thus violating the assumptions of conventional conebeam reconstruction algorithms. Often, these artifacts result in incorrect image information and narrow window values inside the VOI and greatly limit the utility of VOI C-arm CT acquisitions. Thus, they need to be corrected.

Recently, a novel truncation-robust reconstruction algorithm, called Approximated Truncation Robust Algorithm for Computed Tomography (ATRACT), has been proposed in the literature. It is obtained by reformulating the conventional FDK reconstruction method into a scheme that is, by construction, less sensitive to data truncation. ${ }^{12,13}$ The standard FDK reconstruction algorithm consists of 3 steps: 1) preweighting of the projection images, 2) 1D ramp filtering, and 3) a backprojection step. The ATRACT algorithm is based on a decomposition of the conventional ramp filtering (step 2) into a local Laplace filtering and a nonlocal residual filtering. The particular ATRACT variant we applied (2D ATRACT) is based on a 2D Laplace filtering and a 2D convolution-based residual fil- tering, ${ }^{13}$ which can be computed very efficiently, as opposed to the originally proposed $2 \mathrm{D}$ radon-based filtering. ${ }^{12}$

In this article, we propose to evaluate the utility of the VOI acquisition and the accuracy of the ATRACT reconstruction algorithm. Our primary research objective was to determine whether the VOI C-arm CT image acquisition can be incorporated into routine clinical workflow and whether the information discerned from such images can be useful during treatment.

\section{MATERIALS AND METHODS}

VOI C-arm CT images were acquired in 28 patients under an institution-approved protocol. Twenty-five of 28 aneurysms were internal carotid artery aneurysms and were treated by using a flow-diverter device (Pipeline Embolization Device; Covidien, Irvine, California). The remaining 3 aneurysms (2 basilar tip bifurcation aneurysms and 1 anterior communicating artery bifurcation aneurysm) were treated with stents and coils. All the treatments were performed in an interventional suite equipped with a biplane flat panel detector angiographic system (Axiom Artis zee biplane; Siemens AG, Forchheim, Germany). Routine 2D DSA and 3D angiographic images were acquired during the treatment. A noncontrast full-head C-arm CT acquisition (syngo DynaCT Head 20-second protocol; Siemens AG) is routinely acquired as part of our clinical workflow immediately after stent or flow-diverter placement, to examine parenchymal enhancement for ruling out possible hemorrhages.

Additionally, VOI C-arm CT images were acquired in these patients after device deployment. The x-ray source was collimated in both vertical and lateral directions, and the $\mathrm{C}$-arm was isocentered around the deployed stent or flow diverter (Fig 1). The FOV of the VOIC-arm CT acquisitions was set to $11.7 \times 11.7 \mathrm{~cm}$, approximately $12 \%$ of the full FOV $(30 \times 40 \mathrm{~cm})$. VOI C-arm CT images were acquired by using the following parameters: 20 -second rotation, $200^{\circ}$ angular coverage; $0.4^{\circ}$ angular increment; $1240 \times 960$ projection matrix; 496 projections; 30 frames per second; $1.2-\mu \mathrm{Gy} /$ frame sys- 

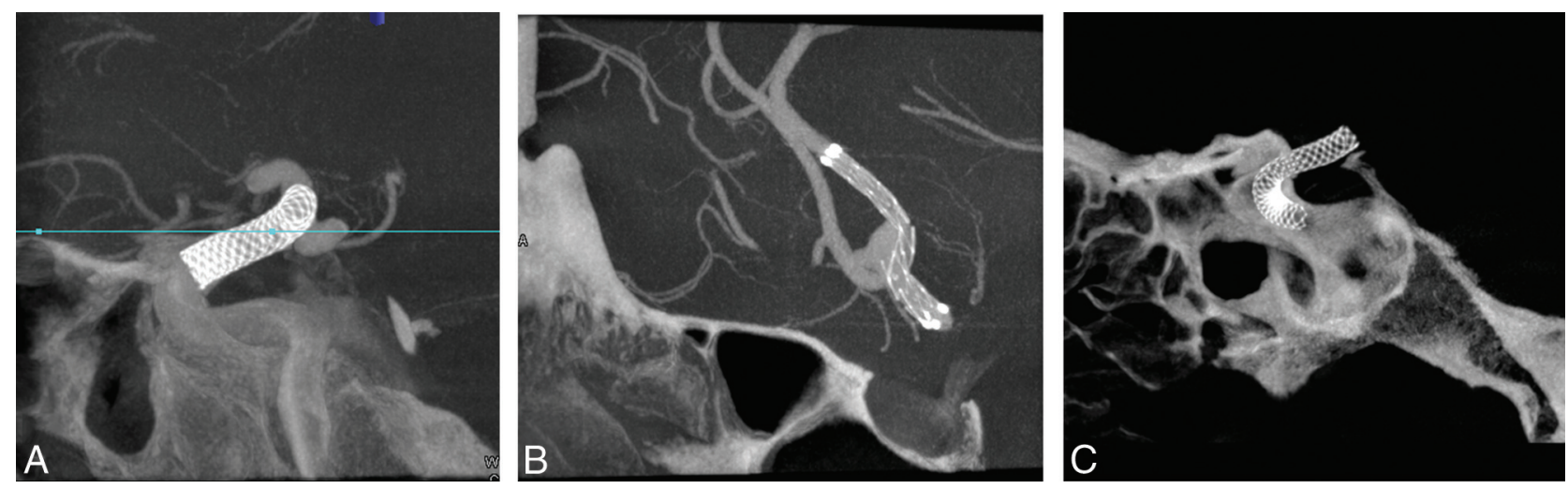

FIG 2. Sample cross-sections from the ATRACT reconstruction of VOI C-arm CT images from the patient cohort. VOI images show clear visibility of stent and flow-diverter devices and their relationship with the parent artery.

tem dose; 48-cm zoom. VOI C-arm CT images were reconstructed with the ATRACT reconstruction algorithm on an off-line workstation, equipped with a Xeon Quad Core processor with a NVIDIA Quadro FX 5800 graphics card (Intel, Santa Clara, California).

Thirty-one VOI images were obtained in 28 patients. Eighteen of 31 VOI C-arm CT images were acquired without contrast injection to assess the reconstruction accuracy of ATRACT. The remaining 13 of 31 VOI C-arm CT images were acquired with contrast medium injected into the internal carotid artery to assess device visibility in VOI images. Iodinated contrast (iohexol, Omnipaque 300; GE Healthcare, Piscataway, New Jersey) was diluted to $20 \%$ concentration with normal saline and injected for 22.5 seconds (20-second rotation time, 2.5-second $\mathrm{x}$-ray delay, $1-\mathrm{mL} / \mathrm{s}$ injection rate, $22.5-\mathrm{mL}$ total volume [ $5-\mathrm{mL}$ of iodinated contrast diluted with 20 -mL of saline]).

One of the main goals of the study was to quantitatively assess the ATRACT reconstruction of VOI images. Historically, medical image quality has been assessed qualitatively by expert raters. However, such comparison is often subject to user error and viewing conditions and requires multiple raters. These shortcomings can be overcome by mathematic formulations that can be computed easily and applied universally. These quantitative measures can be classified into 2 categories consisting of purely mathematically defined measures such as correlation coefficient, mean squared error, and so forth; and the second class incorporating human visual perception of image quality into the formulation.

Because the noncollimated full-head acquisition was also performed on the same patient as part of the routine care, conventional FDK reconstruction of the full-head acquisition serves as a ground truth reference image, facilitating quantitative assessment. We selected 2 numeric metrics, correlation coefficient ${ }^{14}$ and the Structural Similarity Index Metric (SSIM), ${ }^{15}$ to capture the image differences due to the VOI acquisition. The Pearson Correlation Coefficient measures the degree to which both images are similar and is computed by using a simple mathematic formula. Correlation coefficient values range from -1 to +1 . A value of +1 indicates that both images are identical, a value of zero means that images are completely uncorrelated, and a value of -1 means that the images are negatively correlated; for example, the image being compared is an inverse of the reference image.

SSIM also assumes a value between -1 to +1 and was proposed as a method to measure image quality based on an initial uncompressed image as a reference. Compared with other traditional metrics, SSIM also tries to capture perceived visual changes through image degradation. The concept of structural information is derived from the idea that neighboring pixels belonging to a structure have strong interdependencies when they are spatially close and thus define the structure of the objects in the image. In the context of this article, a higher SSIM value (close to 1) indicates that image degradation due to truncation artifacts from the VOI acquisition is eliminated with ATRACT robust reconstruction and that ATRACT also preserves anatomic and device-related information similar to that in the noncollimated acquisition. On the other hand, a low SSIM value indicates poor image quality of VOI C-arm CT images, indicating that the truncation artifacts are still prevalent. Furthermore, a value of 1 is only achievable if both images are identical.

\section{RESULTS}

Excellent visibility of stents and flow-diverter devices was noted in the VOI C-arm CT images in all 28 patients, acquired both with and without contrast medium injection (Fig 2). Stent expansion, wall apposition, and neck coverage were depicted clearly in the VOI C-arm CT images reconstructed with the ATRACT robust reconstruction algorithm. VOI C-arm CT images from conventional FDK reconstructions have severe truncation artifacts, resulting in capping/cupping artifacts and a substantial offset in Hounsfield unit values as seen in Fig 3B. These truncation artifacts were greatly reduced with the ATRACT reconstruction (Fig 3C).

Figure $3 D$ shows a plot of gray-scale intensity values along the colored lines shown on the sample cross-sections in Fig $3 A-C$. The profile of the gray-scale value plot from the ATRACT 2D VOI C-arm $\mathrm{CT}$ reconstruction aligns closely with the conventional FDK reconstruction of the noncollimated full-head C-arm CT acquisition compared with the conventional FDK reconstruction of the VOI C-arm CT images, in which only the high-contrast objects are visualized. A slight misalignment of the gray-scale values is caused by the residual differences because the images were aligned and resampled by using 3D-3D fusion. A slight disparity in the Hounsfield unit values was observed in the line profile graph at the beginning and the end of the line (also seen in Fig $3 \mathrm{C}$ outside the VOI). Indeed, this divergence of Hounsfield unit values was observed in the region outside the VOI being defined by the collimation size used during data acquisition and indicates incorrect reconstruction due to insufficient projection 


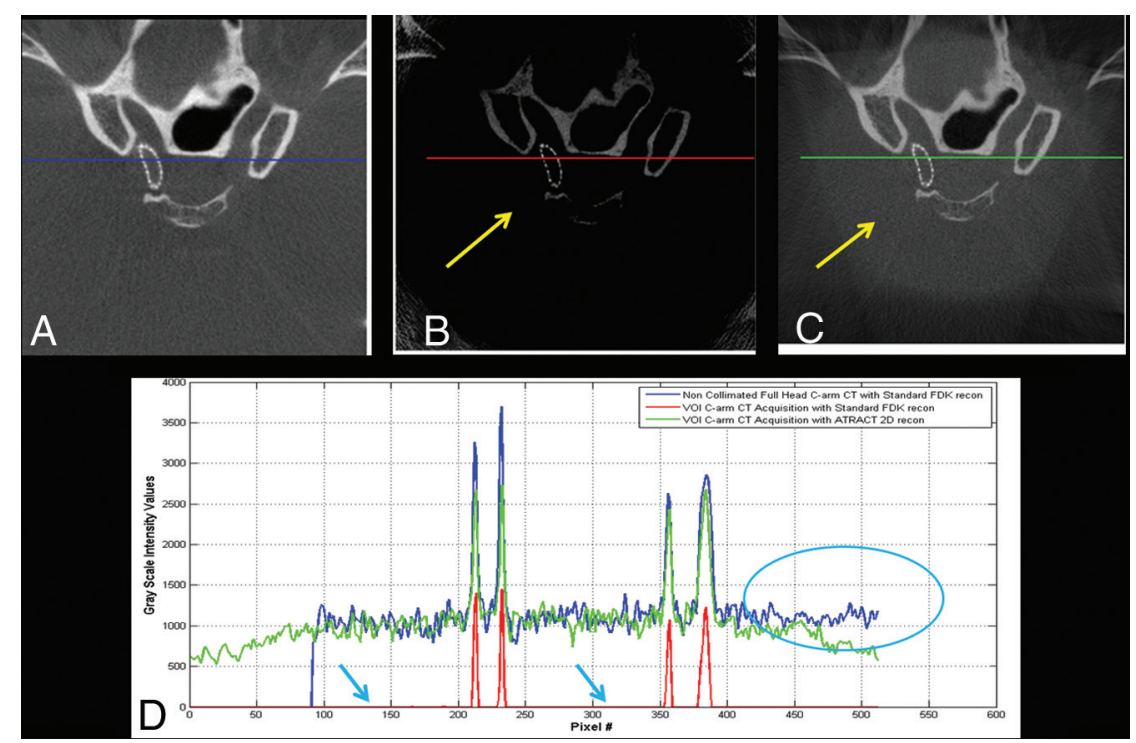

FIG 3. Sample cross-section of a C-arm CT acquisition after Pipeline (flow-diverter) embolization of a right ICA aneurysm. $A$, Cross-section from a secondary reconstruction of a full-head C-arm CT acquisition (also used as a reference image for comparison). B, Crosssection from a standard FDK reconstruction of a VOI C-arm CT acquisition. C, Cross-section from the ATRACT robust reconstruction of the same VOI C-arm CT acquisition. D, Plot of normalized gray-scale values as shown by the colored lines above. Note the information loss from truncation artifacts (see arrows in $B$ and $D$ ) recovered with the ATRACT robust reconstruction algorithm in $C$.

data in the region outside the defined VOI, which is not of diagnostic interest.

Similar conclusions were drawn from quantitative analysis (Fig 4). Similarity metrics showed an improvement in image quality with ATRACT 2D truncation-correction reconstruction (Fig $4)$. The average correlation coefficient was $0.96(\sigma=0.02)$, and the average SSIM was $0.92(\sigma=0.01)$. Moreover, the SSIM value is close to 1 for flow-diverter and other bony landmarks, indicating a very strong image similarity for high-contrast radio-attenuated objects. The average reconstruction time for ATRACT 2D truncation-correction reconstruction is 77.2 seconds compared with 43.5 seconds for the conventional FDK reconstruction of the full-head acquisition. The reconstruction time was measured from the time of initiating the reconstruction to loading the volume into the $3 \mathrm{D}$ workstation and includes the time to write the reconstructed volume to the data base.

Figure 5 shows the dose-area product (DAP) of VOI C-arm CT images and the corresponding noncollimated C-arm CT image from the same patient. An average reduction of $91.5 \%$ in the DAP is observed with the VOI C-arm CT acquisition (mean percentage reduction in $\mathrm{DAP}=91.5 \%$, SD percentage reduction in $\mathrm{DAP}(\sigma)=0.39 \%$, maximum percentage reduction in $\mathrm{DAP}=92.45 \%$ ). Note that these dose reductions were measured in the DAP and may not result in a similar reduction rate in effective dose measurements.

Illustrative Case 1. A 58-year-old woman who presented with a basilar tip bifurcation aneurysm was treated with stents and coils (Fig 6). A noncollimated full-head C-arm CT acquisition (20-second DynaCT protocol) was performed to examine the brain parenchyma immediately after the stent was deployed. Additionally, a 20-second VOI C-arm CT was acquired with dilute contrast injection (injection protocol given above) to study the relationship of the device with the parent artery. Figure $6 B$ shows the ATRACT reconstruction of the VOI acquisition. The stent deployed into the right posterior cerebral artery was well-apposed and provided good neck coverage, thus avoiding a need to deploy a second stent into the left posterior cerebral artery. Furthermore, the stent was manually delineated from the 3D images and overlaid on the live fluoroscopic images for assistance during coiling (Fig 6C). A 91.7\% reduction in DAP values was achieved in this example with the VOI C-arm CT acquisition.

Illustrative Case 2. A 62-year-old woman referred for endovascular treatment of a right internal carotid artery aneurysm was treated with a Pipeline flow-diverter device. Routine clinical images were obtained before and after flow-diverter deployment. A noncollimated full-head C-arm CT acquisition (20-second DynaCT protocol) was performed to examine the parenchyma immediately after the flow diverter was deployed. Malapposition of the flow-diverter device was observed on 2D DSA images and confirmed on the posttreatment Carm CT imaging (Fig 7). Balloon angioplasty was performed, and 20-second DynaCT Head VOI C-arm CT images were acquired with dilute contrast injection (injection protocol given above) to confirm the device apposition after angioplasty. The reconstructed VOI images shown in Fig 7C indicate good apposition of the flow-diverter device to the wall of the internal carotid artery after balloon angioplasty, good coverage of the neck of the aneurysm, and some stasis of contrast medium in the aneurysm. The additional C-arm CT image after angioplasty was acquired with 91\% reduction in the DAP compared with the original full-head acquisition.

\section{DISCUSSION}

C-arm CT imaging has become ubiquitous in the endovascular treatment of intracranial aneurysms. The information gained from C-arm CT imaging enables the operator to assess the treatment, make informative choices, and take corrective measures accordingly during the procedure. However, multiple C-arm CT acquisitions can contribute substantially toward cumulative radiation exposure to the patient. A new imaging technique, VOI $\mathrm{C}$-arm CT imaging, is presented, which acquires C-arm CT images of only the ROI at a reduced dose.

VOI C-arm CT imaging uses extensive collimation in both horizontal and vertical directions, thus achieving dose savings by avoiding radiation exposure to anatomic regions that are outside the ROI. However, collimation results in severe truncation artifacts. Thus, we evaluated a novel truncation-robust reconstruction algorithm, 2D ATRACT, which reduces truncation artifacts from the VOI acquisition. Before ATRACT, various techniques to reconstruct meaningful VOI C-arm CT images with minimal truncation artifacts have been proposed in the literature. The common theme of these 

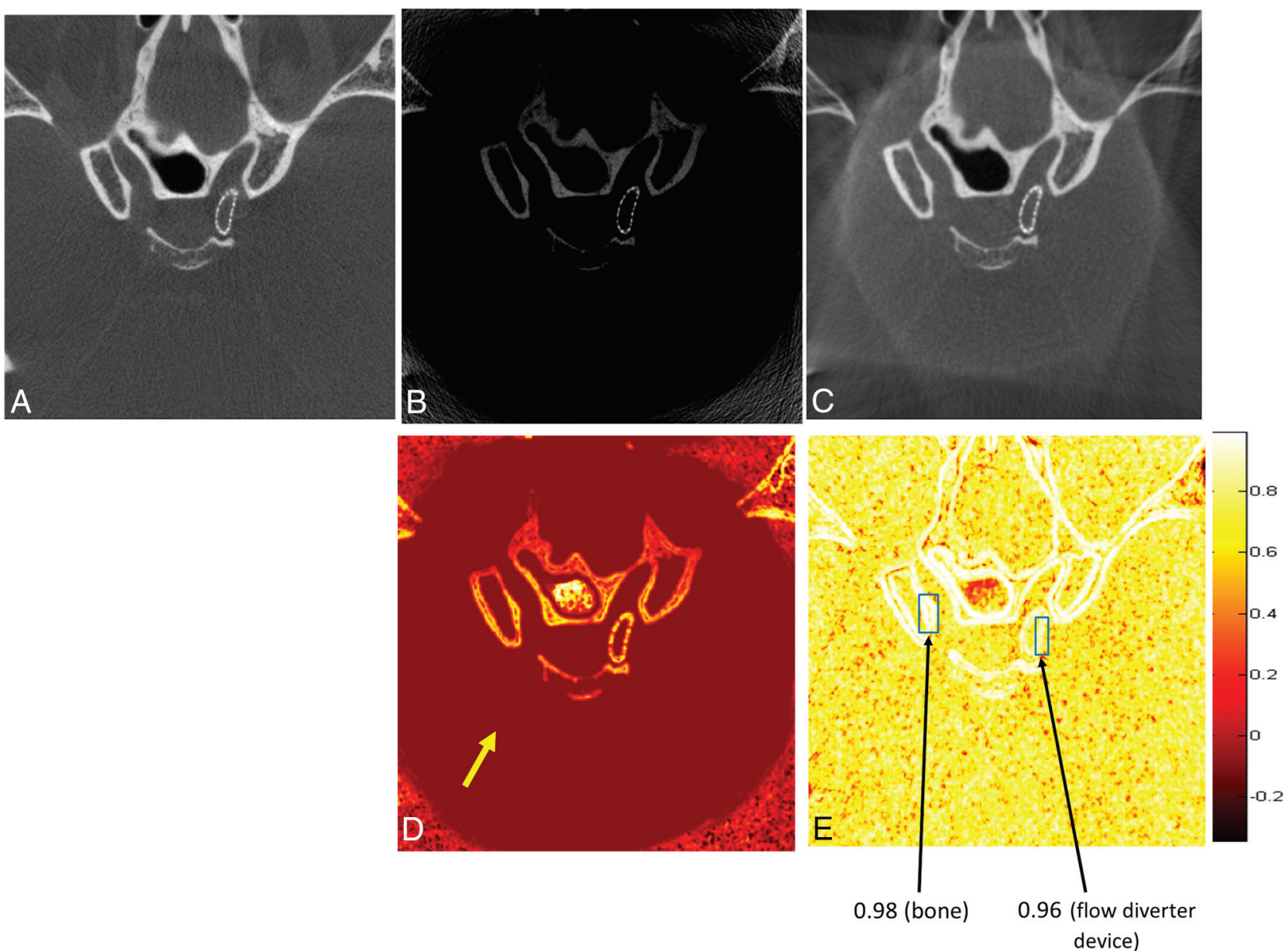

FIG 4. Quantitative comparison of image quality of the VOI C-arm CT acquisition by using the Structural Similarity Index Metric. A, Cross-section of a secondary reconstruction from a full-head C-arm CT acquisition. $B$, Cross-section from a standard FDK reconstruction of a VOI C-arm CT acquisition. $C$, Corresponding cross-section from an ATRACT reconstruction of the VOI C-arm CT acquisition. D, SSIM map of $B$ and A. E, SSIM map of $C$ and $A$. Each pixel in $D$ and $E$ represents $S S I M$ values ranging from -1 to +1 . $A$, This image is used as the ground truth reference image; the VOI acquisition is aligned with the full-head acquisition to establish voxel-to-voxel correspondence. Note the improvement in SSIM values corresponding to soft tissue before and after truncation correction as shown by the yellow arrow in D. Bony landmarks and devices have SSIM values close to 1, indicating a strong similarity between the ATRACT 2D VOI reconstruction and the standard FDK full-head reconstruction as shown by arrows in $E$.
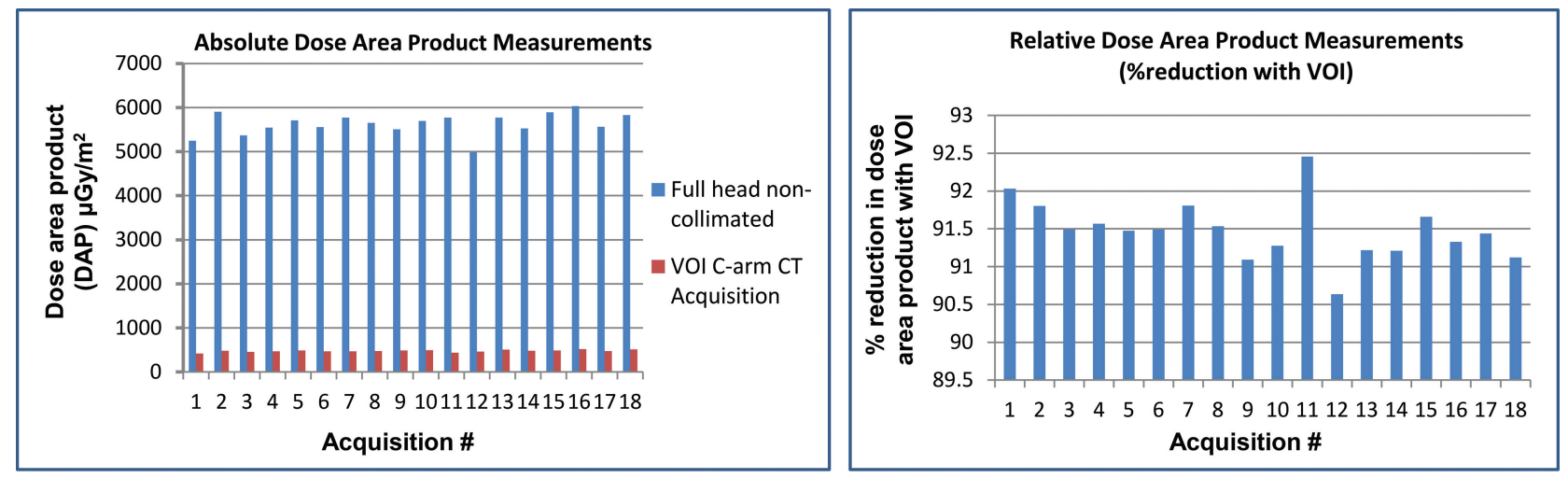

FIG 5. Comparison of an absolute and relative dose-area product between the noncollimated full-head C-arm CT acquisition and the collimated VOI C-arm CT acquisition. Note that these measurements are from the same patient for each study. An average reduction of $91.45 \%$ in the DAP is observed with the VOI acquisition.

techniques is to fill in the missing information either by using preinterventional $\mathrm{CT},{ }^{16}$ extrapolating missing data on the basis of some heuristic approach, ${ }^{17}$ acquiring a low-resolution full-head scan and combining it with truncated VOI projection data with full-field data to create a full projection matrix suit- able for a FDK reconstruction algorithm, ${ }^{18}$ or using advanced filtering algorithms to eliminate truncated data. ${ }^{19,20}$

Because most of these methods were designed for a stationary $\mathrm{C}$-arm gantry and a rotating object and less robust extrapolation techniques, their utility for neurointerventional applications is 

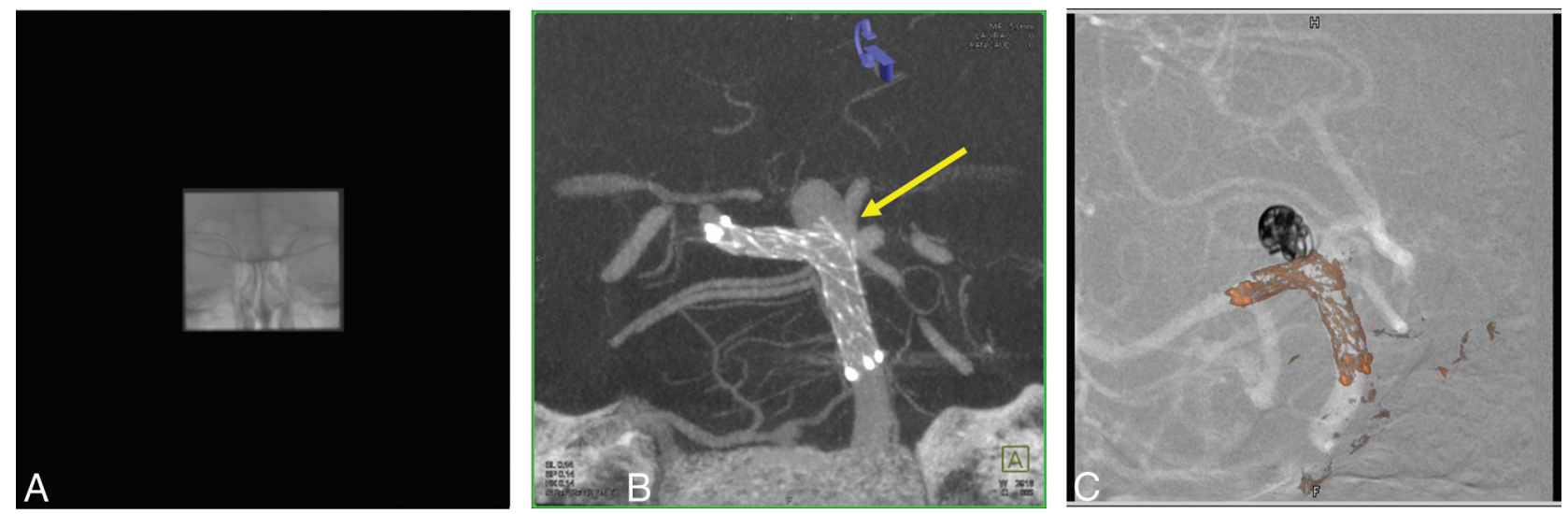

FIG 6. VOI C-arm CT images of a basilar tip aneurysm in a 58-year-old woman treated with stent and coils. Contrast-enhanced VOI C-arm CT images were acquired immediately after the stent was deployed. A, Frontal projection of a VOI C-arm CT acquisition. B, ATRACT reconstruction of the same VOI C-arm CT. C, Fluoroscopic overlay of the stent for assistance during coiling. Note that the high-contrast objects like stents are well-visualized with the VOI C-arm CT images. In this particular example, based on the C-arm CT imaging neck coverage was good after the deployment for first stent from the right side, thus avoiding the need for a second stent.
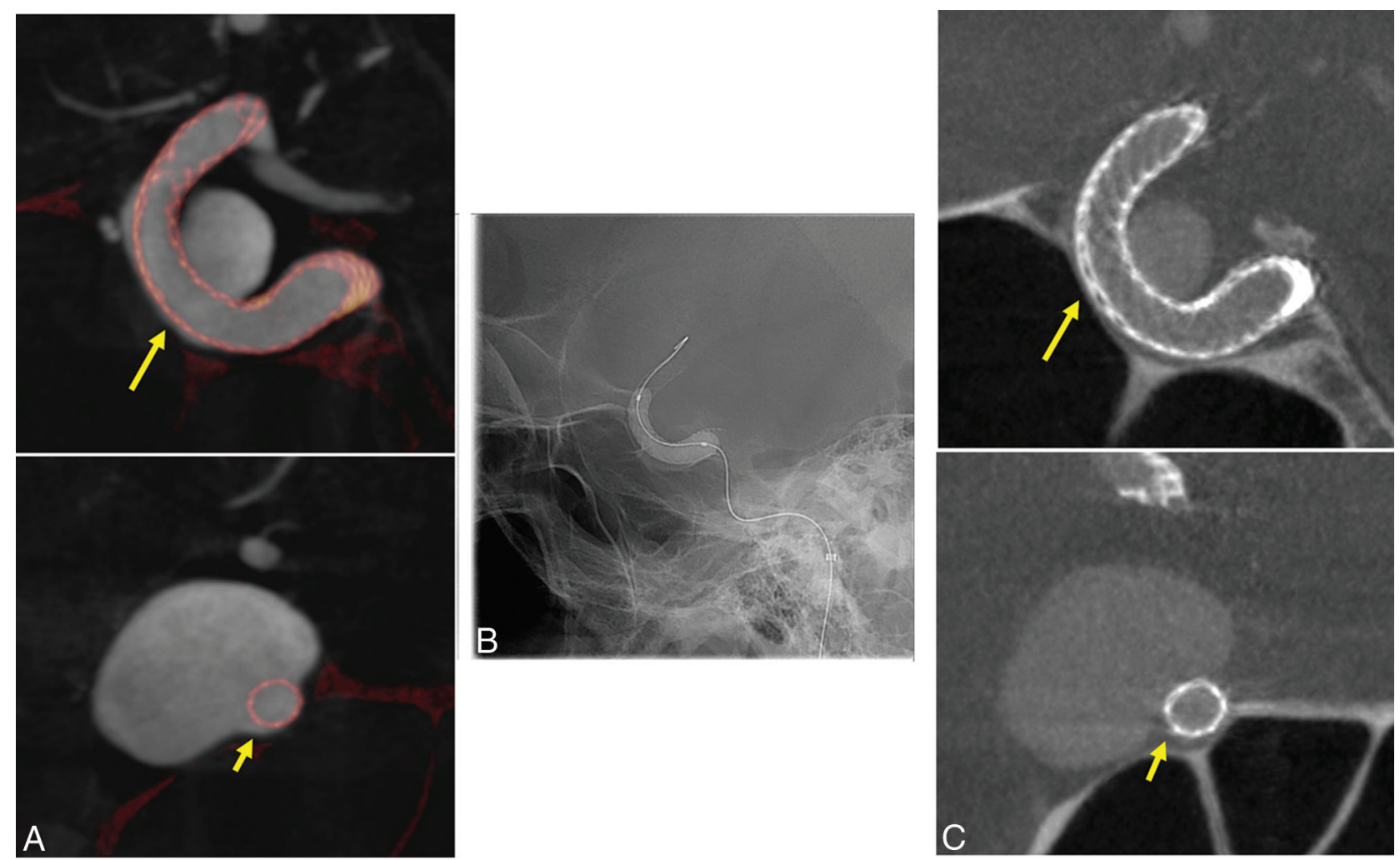

FIG 7. Sample $C$-arm CT cross-sectional images of an ICA aneurysm in a 62-year-old woman treated with the Pipeline (flow-diverter) Embolization Device. $A$ and $B, C$-arm CT images postdeployment show the malapposition of the flow-diverter device to the parent artery. $B, 2 D$ fluoroscopic image shows the balloon angioplasty fully expanding the flow-diverter device. C, Cross-sections from the ATRACT VOI C-arm CT images show improved wall apposition of the flow-diverter device to the parent artery.

very limited. Kolditz et $\mathrm{al}^{21}$ developed a technique to obtain a sparse overview scan and a high-resolution VOI scan. However, the sparse overview scans add dose and time and impose registration constraints between the overview and the VOI scan. Patel et $\mathrm{al}^{22}$ explored a similar technique by mounting a microangiographic fluoroscope onto the regular angiography equipment to preserve the small details required for neuroradiologic applications. Similar techniques that use dual detectors have been proposed for breast imaging applications that can be extended to neuroradiology applications. ${ }^{23}$
In contrast, ATRACT is based on an analytic reformulation of the standard FDK algorithm into a reconstruction scheme that is, by construction, less sensitive to lateral data truncation. Even in the presence of severe lateral data truncation, the algorithm effectively reduces truncation artifacts and provides high-quality reconstructions without explicit extrapolation or prior knowledge, thus making it clinically feasible. ${ }^{12,13}$

The clinical examples presented in this article illustrate the added value of VOI C-arm CT imaging. The VOI imaging technique provides clear visualization of stents and flow-diverter de- 
vices and their relationship with the parent artery. Quantitative analysis showed a strong correlation between truncation-corrected VOI images and noncollimated C-arm CT images. High contrast objects, such as metallic implants, showed relatively higher correlation compared with the soft-tissue information. For quantitative comparison, images were aligned by using 3D-3D fusion because VOI C-arm CT images were acquired with the device at the isocenter and the $\mathrm{C}$-arm position was different for full-head and VOI C-arm CT acquisitions. The resulting resampling from 3D-3D fusion could have potentially contributed to the discrepancies in image similarity metrics.

During the acquisition of VOI C-arm CT images, it is very crucial to isocenter the $\mathrm{C}$-arm around the deployed device. Depending on the type of device (stent markers and coils are easily visible on fluoroscopic imaging compared with the flow-diverter device in the cavernous segment surrounded by bony anatomy), it is often difficult to isocenter the $\mathrm{C}$-arm and multiple fluoroscopic $\mathrm{X}$-ray images were required to visualize the device, especially with a small VOI, because there are not many landmarks to be seen in the ROI. Automatic isocentering based on a sparse low-dose overview scan or pretreatment $3 \mathrm{D}$ images or $2 \mathrm{D}$ biplane angiographic images during treatment is desirable. Given that the dose savings are proportional to the size of the VOI, advanced algorithms to automatically detect the ROI and to automatically identify the size of the VOI are needed to further seamlessly integrate VOI imaging into clinical workflow.

\section{CONCLUSIONS}

VOI C-arm CT imaging with a new truncation-robust reconstruction algorithm allows acquisition of multiple intraprocedural C-arm CT images with a relatively lower additional dose delivered to the patient. VOI C-arm CT images provide devicespecific information about its relationship with the parent artery during the procedure, thus resulting in a valuable tool to assess treatment efficacy and allow additional corrective measures in case of inaccurate deployment.

Disclosures: Gouthami Chintalapani-UNRELATED: Employment: full-time employee at Siemens Medical Solutions USA. Ponraj Chinnadurai-UNRELATED: Employment: Siemens Medical Solutions USA Inc, Comments: employed as a full-time senior staff scientist, Angiography Division, Siemens Medical Solutions USA, Hoffman Estates, Illinois. Andreas Maier-RELATED: Grant: Siemens is supporting the research project of Yan Xia at the University of Erlangen. Yan Xia is now working under my supervision towards his PhD on VOI reconstruction algorithms, which are used in this article*; Consulting Fee or Honorarium: I have been a researcher at Siemens AG, and I developed parts of the algorithms that are used in this work as a Siemens employee; UNRELATED: Employment: I have been a researcher at Siemens AG; Grants/Grants Pending: Siemens is supporting several of my research projects*; Patents (planned, pending or issued): I have several patents with Siemens AG. Sebastian Bauer-UNRELATED: Employment: Siemens AG, Forchheim, Germany. *Money paid to the institution.

\section{REFERENCES}

1. Benndorf G1, Ionescu M, Y Alvarado MV, et al. Wall shear stress in intracranial self-expanding stents studied using ultra-high-resolution 3D reconstructions. AJNR Am J Neuroradiol 2009;30:479-86 CrossRef Medline

2. Ionita C, Natarajan S, Wang W, et al. Evaluation of a second-generation self-expanding variable-porosity flow diverter in a rabbit elastase aneurysm model. AJNR Am J Neuroradiol 2011;32:1399407 CrossRef Medline
3. Makoyeva A, Bing F, Darsaut T, et al. The varying porosity of braided self-expanding stents and flow diverters: an experimental study. AJNR Am J Neuroradiol 2013;34:596-602 CrossRef Medline

4. Akpek S, Brunner T, Benndorf G, et al. Three-dimensional imaging and cone beam volume $\mathrm{CT}$ in $\mathrm{C}$-arm angiography with flat panel detector. Diagn Interv Radiol 2005;11:10-13 Medline

5. Benndorf G, Strother CM, Claus B, et al. Angiographic CT in cerebrovascular stenting. AJNR Am J Neuroradiol 2005;26:1813-18 Medline

6. Doelken M, Struffert T, Richter G, et al. Flat-panel detector volumetric CT for visualization of subarachnoid hemorrhage and ventricles: preliminary results compared to conventional CT. Neuroradiology 2008;50:517-23 CrossRef Medline

7. Heran NS, Song JK, Namba K, et al. The utility of DynaCT in neuroendovascular procedures. AJNR Am J Neuroradiol 2006;27: 330-32 Medline

8. Kizilkilic O, Kocer N, Metaxas GE, et al. Utility of VasoCT in the treatment of intracranial aneurysm with flow-diverter stents. J Neurosurg 2012;117:45-49 CrossRef Medline

9. Kamran M, Nagaraja S, Byrne JV. C-arm flat detector computed tomography: the technique and its applications in interventional neuro-radiology. Neuroradiology 2012;52:319-27 CrossRef Medline

10. Fiorella D, Hsu D, Woo HH, et al. Very late thrombosis of a Pipeline embolization device construct: case report. Neurosurgery 2010;67(3 suppl operative):E313-14 CrossRef Medline

11. Struffert T, Hauer M, Banckwitz R, et al. Effective dose to patient measurements in flat-detector and multislice computed tomography: a comparison of applications in neuroradiology. Eur Radiol 2014;24: 1257-65 CrossRef Medline

12. Dennerlein F, Maier A. Approximate truncation robust computed tomography: ATRACT. Phys Med Biol 2013;58:6133-48 CrossRef Medline

13. Xia X, Hofmann H, Dennerlein F, et al. Towards clinical application of a Laplace operator-based region of interest reconstruction algorithm in C-arm CT. IEEE Trans Med Imaging 2014;33:593-606 CrossRef Medline

14. Rodgers J, Nicewander W. Thirteen ways to look at the correlation coefficient. Am Stat 1988;41:59-66

15. Wang Z, Bovik AC, Sheikh HR, et al. Image quality assessment: from error visibility to structural similarity. IEEE Trans Image Process 2004;13:600-12 CrossRef Medline

16. Wiegert J, Bertram M, Netsch T, et al. Projection extension for region of interest imaging in cone-beam CT. Acad Radiol 2005;12: 1010-23 CrossRef Medline

17. Hsieh J, Chao E, Thibault J, et al. A novel reconstruction algorithm to extend the CT scan field-of-view. Med Phys 2004;31:2385-91 CrossRef Medline

18. Chityala R, Hoffmann KR, Rudin S, et al. Region of interest (ROI) computed tomography $(\mathrm{CT})$ : comparison with full field of view (FFOV) and truncated CT for a human head phantom. Proc SPIE Int Soc Opt Eng 2005;5745:583-90 Medline

19. Cho S, Bian J, Pelizzari CA, et al. Region-of-interest image reconstruction in circular cone-beam microCT. Med Phys 2007;34: 4923-33 CrossRef Medline

20. Yu L, Zou Y, Sidky EY, et al. Region of interest reconstruction from truncated data in circular cone-beam CT. IEEE Trans Med Imaging 2006;25:869-81 CrossRef Medline

21. Kolditz D, Struffert T, Kyriakou Y, et al. Volume-of-interest imaging of the inner ear in a human temporal bone specimen using a robotdriven C-arm flat panel detector CT system. AJNR Am J Neuroradiol 2012;33:E124-28 CrossRef Medline

22. Patel V, Hoffmann KR, Ionita CN, et al. Rotational micro-CT using a clinical C-arm angiography gantry. Med Phys 2008;35:4757-64 CrossRef Medline

23. Shen Y, Yi Y, Zhong Y, et al. High resolution dual detector volumeof-interest cone beam breast CT: demonstration with a bench top system. Med Phys 2011;38:6429-42 CrossRef Medline 